\title{
Droplets, vapours and clouds - a new approach to capacitive transducer manufacture
}

\author{
Richard L O’Leary \& Gordon Brown \\ Dept of Electronic and Electrical Engineering \\ University of Strathclyde, \\ Glasgow, UK
}

\author{
Gerry Harvey \\ PZFlex, Weidlinger Associates \\ Glasgow, UK
}

\begin{abstract}
Capacitive ultrasonic transducers (cUT) comprise a substrate patterned with a regular array of uniformly dimensioned cavities above which a membrane is positioned. Transmission and reception of ultrasound is effected via controlled electrical or mechanical stimulus of the membrane, respectively. The dimensions of the cavity and the mechanical properties of the membrane determine the vibrational behavior of the resultant transducer.
\end{abstract}

This paper employs a facile process for the micropatterning of polymer substrates for the manufacture of capacitive transducers. A positive mask of the desired cavity microstructure is deposited onto a polystyrene substrate. The substrate is then exposed to a saturated toluene vapour which is absorbed by the polystyrene causing swelling of the surface except in the areas where the droplets are situated resulting in the formation of micro-cavities at the position of each droplet. The PZFlex finite element (FE) software, deployed on custom cloud computing architecture allowing for 1000's of simultaneous parallel simulations, has been employed to explore the potential for the use of the micropatterned substrates in the manufacture of capacitive ultrasonic transducers. This new cloud approach has facilitated reducing months of intensive FE modelling to a few days.

The results have been employed to guide the optimisation of the patterning process in order to manufacture devices suited to aircoupled non-destructive testing. The paper describes the results of the finite element modelling, the optimisation of the manufacturing route and the characterisation of the resultant devices.

Keywords-capacitive transducer; modelling; finite element; aircoupled ultrasound

\section{INTRODUCTION}

Capacitive ultrasonic transducers (cUT) comprise a substrate patterned with a regular array of uniformly dimensioned cavities above which a membrane is positioned. Transmission and reception of ultrasound is effected via controlled electrical or mechanical stimulus of the membrane, respectively. The dimensions of the cavity and the mechanical properties of the membrane determine the vibrational behavior of the resultant transducer.

Cavity manufacture can be affected by a wide variety of techniques. From the simple polishing [1]; micro-machining of silicon [2], [3]; microstereolithography (MSL) [4] or the multi- user MEMS process (mumps) [5]. In any of these techniques the goal is the creation of a regular array of cavities with uniform dimensions, geometry and spatial arrangement. Each of the reported techniques has its own relative merits and inherent limitations and there is no panacea for the facile creation of cUT devices across a wide range of length scales.

This paper employs new facile process for the micropatterning of polymer substrates [6] for the manufacture of capacitive devices. A positive mask of the desired cavity microstructure is deposited onto a polystyrene substrate as droplets of ethylene glycol solution. The substrate is then exposed to a saturated toluene vapour which is absorbed by the polystyrene causing swelling of the surface except in the areas where the droplets are situated resulting in the formation of micro-cavities at the position of each droplet. The lateral dimensions of the cavities are controlled by the diameter of the deposited droplets. Cavity depth is directly proportional to the time the sample is exposed to the toluene vapour. Importantly, cavity diameter and depth can be controlled independently therefore the methodology has the potential to create a wide array of devices. In order to explore the range of potential device configurations the PZFlex finite element (FE) code has been employed using a a custom cloud computing architecture allowing for 100's of simultaneous parallel simulations, has been employed to explore the problem space.

In the next Section the manufacture and characterization of cavities within a polymer substrate will be presented. This will be followed by results from the FE modeling exercise - a number of microstructures optimized for air-coupled operation will be presented. Finally, example devices will be presented.

\section{METHODOLOGY}

Polystyrene, 3mm sheet (Amari Plastics, Glasgow), was cut to the desired dimensions and cleaned via sonication in methanol (Sigma Aldrich, Dorset, UK) and air dried at room temperature. Solutions of ethylene glycol (Sigma Aldrich, Dorset, UK) $40 \% \mathrm{v} / \mathrm{v}$ in water were prepared. Two methods of depositing droplets of the ethylene glycol solution onto the polystyrene substrate were investigated. A piezoelectric droplet generator, this allows for the deposition of monodisperse droplets on to the substrate and an air-pressured atomiser can be employed - the latter deposits multiple droplets with a wide distribution of diameter in one operation. Each of these methods will be described in turn and as will be shown in 
Section V, both methods were found to be appropriate for the production of structures suited to air-coupled capacitive transducers.

\section{A. Piezoelectric Droplet Generator}

A droplet generator was manufactured using a piezoelectric tube actuator, (PT120, PI, Germany) fitted with a continuous glass capillary [7]. One end of the glass capillary was shaped by heating in a propane flame to create a $150 \mu \mathrm{m}$ diameter nozzle - this was subsequently polished using $1 \mu \mathrm{m}$ alumina to create a flat surface at the outlet [8]. Figure 1 details a photomicrograph of the nozzle. The glass capillary was bonded into the piezoelectric actuator tube using Araldite Rapid epoxy (Huntsman, Cambridge, UK).

In order to generate droplets the actuator was excited using a -300V impulse, a single pulse resulted in droplet of $120 \mu \mathrm{m}$ being deposited onto the polystyrene substrate as shown in Figure 2 (a). Larger droplet diameters were achieved by depositing multiple droplets in the same location, Figure 2(b)(d), allowing for control of the diameter of individual cavities in the polystyrene substrate. The diameter of the deposited droplet does not increase monotonically with the number of droplet; this is a result of the wetting of the droplet to the polystyrene surface.

\section{B. Aerosol}

Deposition of droplets using an aerosol was also investigated. A reusable air-pressured aerosol bottle (Fisher Scientific, Loughborough, UK) was charged with ethylene glycol solution and pressurized to 2.5 psi using the integral pump. The aerosol generated a fine mist of randomly sized droplets onto the polystyrene substrate. Figure 3 details a photomicrograph of a sample of polystyrene with aerosol generated droplets. The diameters observed in Figure 3 are in the range $30-200$ microns

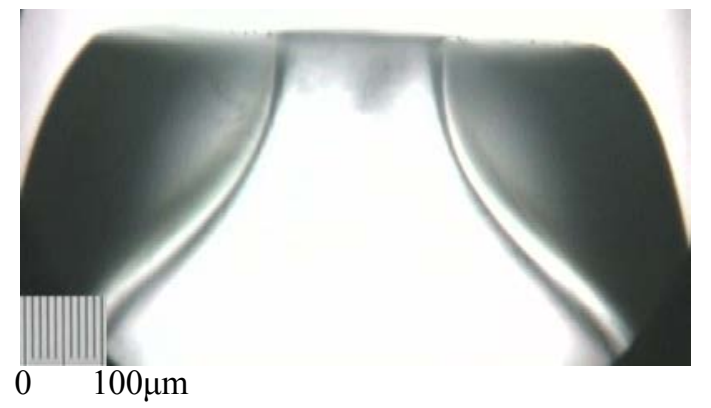

Figure 1. Photomicrograph of the tip of the glass capillary

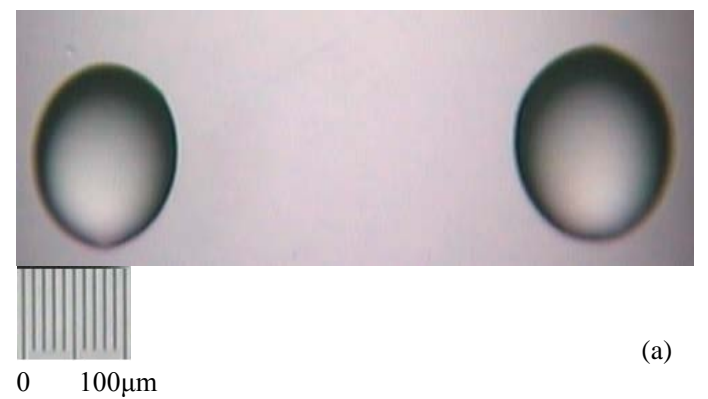

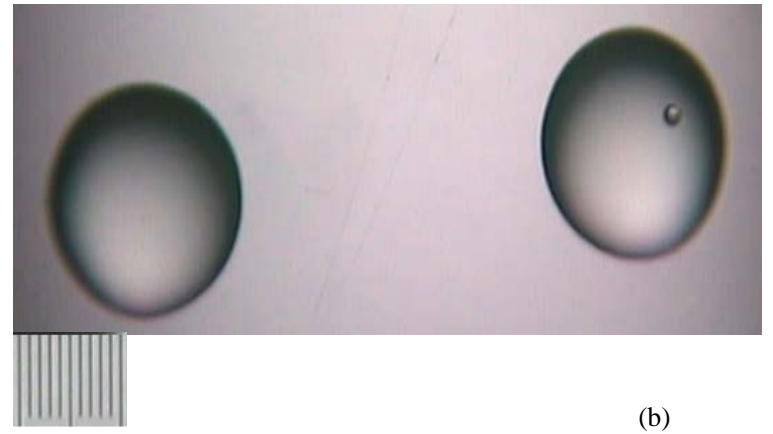

$0 \quad 100 \mu \mathrm{m}$

(b)

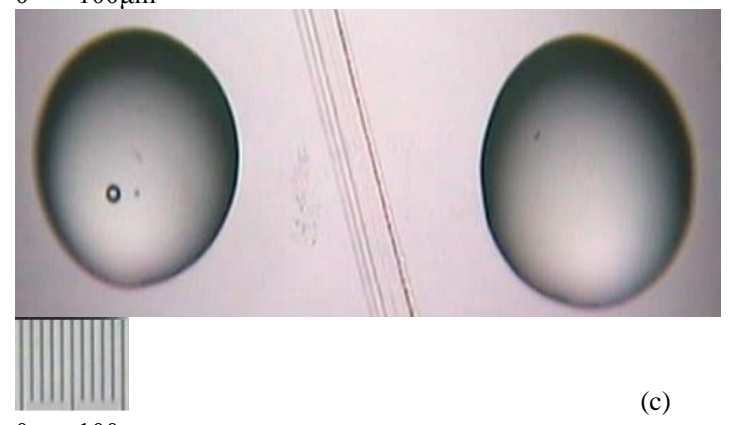

$0 \quad 100 \mu \mathrm{m}$

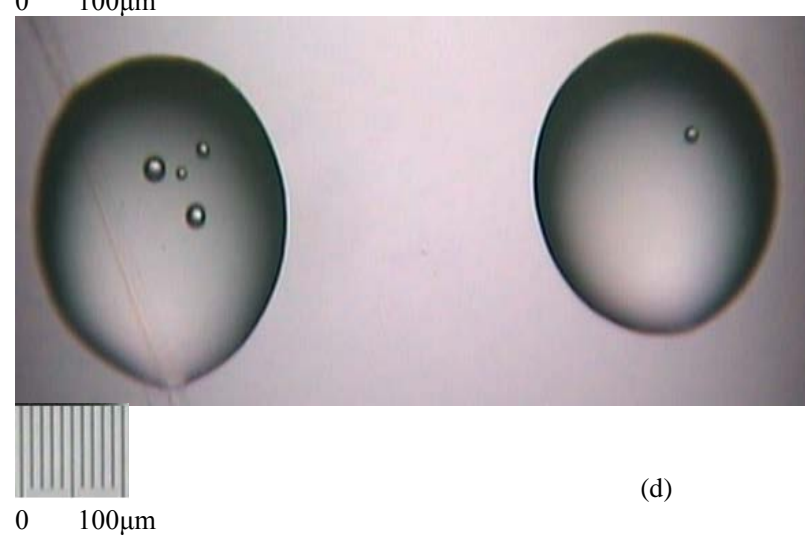

Figure 2. Photomicrographs of droplets of ethylene glycol solution despsited onto polystyrene substrate:

(a) 1 drop per location, (b) 2 drops per location,

(c) 3 drops per location, (d) 4 drops per location

\section{Cavity Formation}

Once the droplets of ethylene glycol solution had been deposited onto the polystyrene the sample was exposed to a vapor of toluene, during this process the polystyrene substrate absorbs toluene causing it to swell. The regions of polystyrene under the deposited droplets do not absorb toluene and hence the do not swell thus forming small cavities within the surface of the polystyrene sample. As will be shown in the next section, the period of time the sample is exposed to the toluene vapor governs the extent of the swelling and hence depth of the cavity. After exposure to toluene vapor, the sample is air dried at $20^{\circ} \mathrm{C}$ and then washed by sonication in deionized water and further air-drying at $20^{\circ} \mathrm{C}$. 


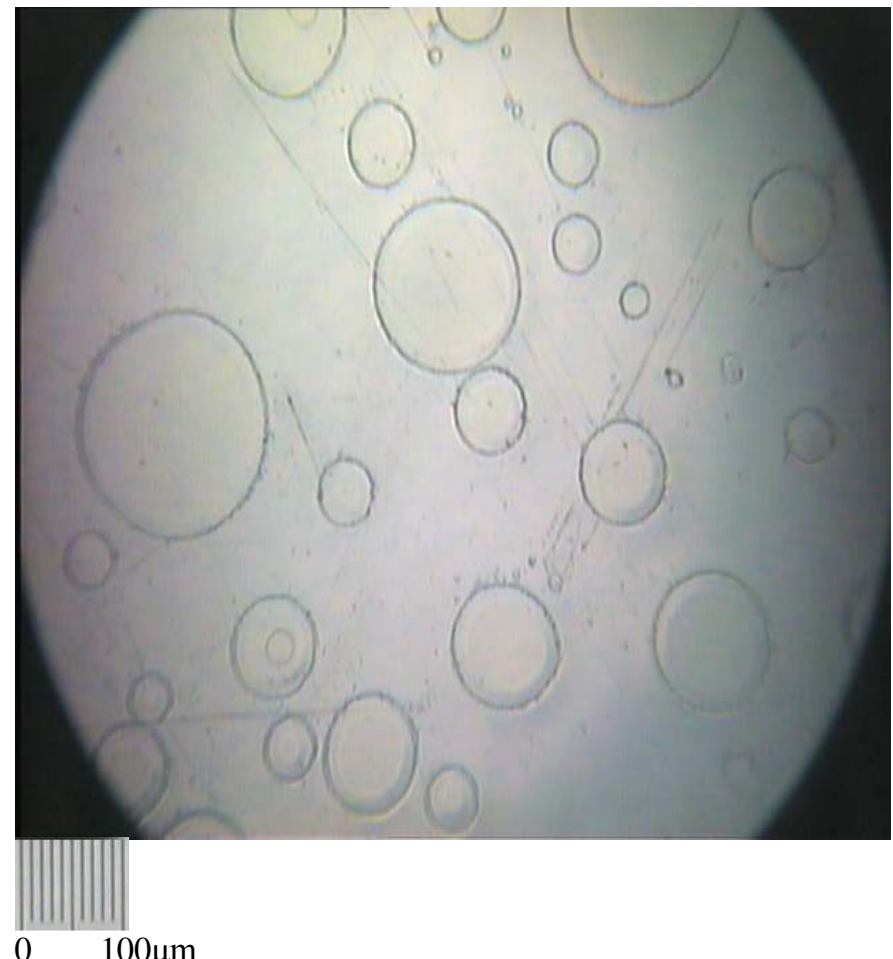

Figure 3. Photomicrograph of aerosol deposited drops

\section{CAVITY CHARACTERISATION}

The cavities were characterized using a Veeco NT1100 optical profiler. This instrument provides a rapid, non-contact method of measuring the height profile of an area (up to a few $\mathrm{mm} 2$ ) using white light interferometry with a resolution in the vertical axis of $0.1 \mathrm{~nm}$. Figure 4 illustrates the variation in cavity depth as a function of the length of time the patterned sample is exposed to the toluene vapor. The data show a nonlinear variation as a function of exposure time. However, the depth of the cavities observed here differ somewhat from those observed by elsewhere [6], this may be a result of the specific grade of polystyrene employed in this study. In the samples that were patterned using the aerosol method, cavity depth was measured for the wide range of cavity diameters observed. The cavity depth was found to be independent of cavity diameter. In addition to depth, the surface roughness of the samples was also assessed. The RMS surface roughness of the polystyrene surface that was exposed to toluene vapor was found to be $10 \mathrm{~nm}$ irrespective of the exposure time. The RMS surface roughness of the floors of the resultant cavities was also found to be $10 \mathrm{~nm}$.

The range of dimensions achievable using the described methodology was used employed in a PZFlex model to explore the potential for the use of the micro-patterned substrates in the manufacture of capacitive ultrasonic transducers.

\section{PZFLEX MODELLING}

Capacitive Ultrasonic Transducers represent a class of problem that is both highly coupled and nonlinear in nature. For this reason, the use of modern $\mathrm{FE}$ analysis has proven effective to model the overall behavior of these systems. However, due to the large number of factors that can affect device key performance metrics, using FE in a traditional hardware platform to capture the necessary design space can be computationally and resource intensive.

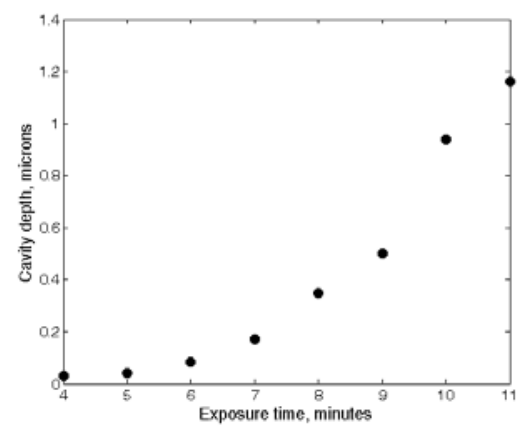

Figure 4. Cavity depth as a function of exposure time

Given this problem, PZFlex has now been deployed in a custom cloud computing architecture (PZFlexCloud), which facilitates the setup of 100's of simultaneous models in a massively parallel arrangement. Figure 5 illustrates one such KPI in pressure spectra vs cavity depth for both $500 \mu \mathrm{m}$ and $1000 \mu \mathrm{m}$ cavity width.

The design space was configured for variations in cavity width, depth and bias voltages to give a total of 1890 unique simulations. Implementing this arrangement on PZFlexCloud delivers the complete dataset in $1 \mathrm{hr}$, requiring $1400 \mathrm{hrs}$ total computational time. This represents a speed-up of approximately 14,000\% over traditional sequential sweeps.

Rapid access to key metrics in this manner provides an intuitive visualization of the large design space and helps narrow parameters of interest for fabrication in future experiments, driving down the overall development time.
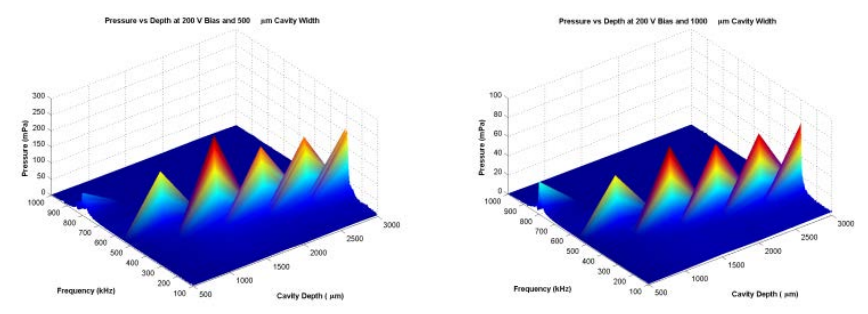

Figure 5. Pressure spectra vs depth for widths $500 \mu \mathrm{m}$ and $1000 \mu \mathrm{m}$

\section{AIR-COUPLED TRANSDUCERS}

The results of the PZFlex modeling were used to guide the manufacture of capacitive devices operating in the sub- $\mathrm{MHz}$ frequency range for air-coupled inspection. Two example devices were manufactured using this methodology; Table 1 details the constructional parameters of two example devices that will be described. In each of the example devices, the piezoelectric actuated droplet generator was used to deposit uniform droplets onto 30mm diameter sample of polystyrene. A Colinbus profiler (Colinbus, Belgium) was used to move the sample of polystyrene in a 2D plane such that constant droplet pitch could be achieved ensuring a uniform spatial distribution of droplets and hence cavities. Once the droplet pattern had been deposited, the polystyrene sample was exposed to toluene 
vapor to create the desired cavity depth in line with the data in Figure 4. After the solvent exposure, washing and drying, the polystyrene substrate is then electroded on all faces with silver using an electroless process with a tin sensitizer [9]. The micropatterned polystyrene was then fitted with 8 micron Kapton membrane (Goodfellow, Huntingdon, UK) the outer face of the dielectric membrane has a sputtered aluminum electrode. Figure 6 shows a photograph of a completed device.

TABLE I. CONSTRUCTIONAL PARAMETERS OF EXAMPLE DECVIES

\begin{tabular}{|l|c|c|}
\hline & Device A & B \\
\hline $\begin{array}{l}\text { Cavity diameter, } \\
\text { microns }\end{array}$ & 400 & 1000 \\
\hline $\begin{array}{l}\text { Target cavity depth, } \\
\text { microns }\end{array}$ & 1.2 & 1 \\
\hline $\begin{array}{l}\text { Actual cavity depth, } \\
\text { microns }\end{array}$ & 1.2 & 0.9 \\
\hline Cavity pitch, microns & 1000 & 1800 \\
\hline
\end{tabular}

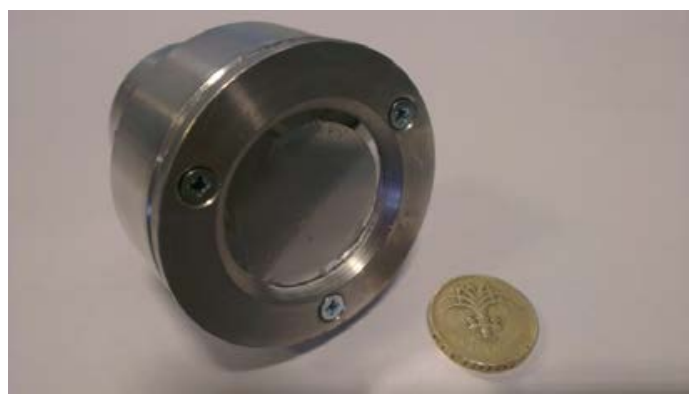

Figure 6. Photgraph of a completed device

The performance of the two devices was assessed using air coupled pulse echo response from a 50mm glass block. In each case a $200 \mathrm{~V}$ bias and impulse excitation was applied to the device. The time and frequency domain data for devices $\mathrm{A}$ and B are shown in Figure 7.
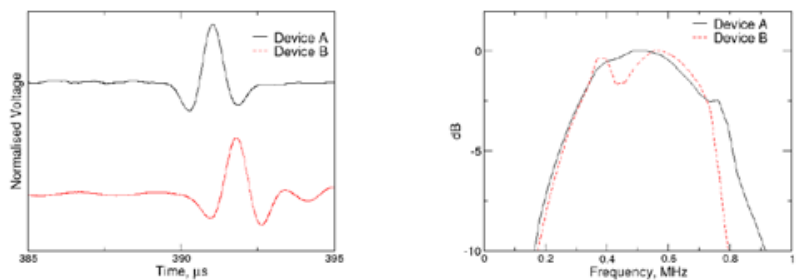

Figure 7. Experimtal air-coupled impulse response data:

(a) time domain data and (b) frequency domain data)

Finally laser vibrometry was performed using an OFV 056 (Polytec, Germany) to determine the surface dilation of the membrane, the magnitude and phase of the surface dilation for device A is shown in Figure 8. The peaks in the magnitude data correspond to each individual cavity - importantly the phase response is uniform
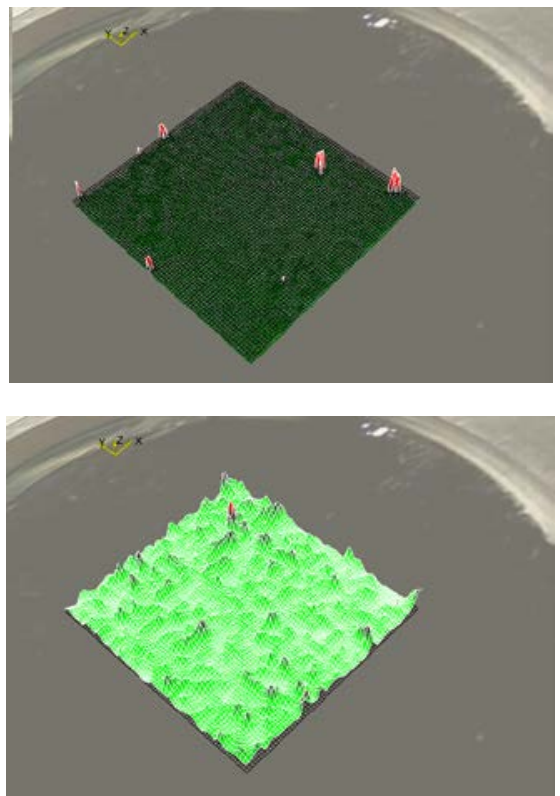

Figure 8. Surface dilation of the membrane for device A

\section{CONCLUDING REMARKS}

A new facile process for micro-patterning polymer substrates for capacitive devices has been investigated. Results for operation in air and a new FE methodology to rapidly explore design space are presented. Future work will consider extending performance for broadband operation in immersed applications.

\section{REFERENCES}

[1] A. Gachagan, et al "Characterization of Air-Coupled Transducers" IEEE Trans Ultrasonics, Ferroelectrics and Frequency Control, Vol 43, No, 4, pp678-689, (1996)

[2] D.W. Schindel, et al "The design and characterization of micromachined air-coupled capacitance transducers" IEEE Trans Ultrasonics, Ferroelectrics and Frequency Control Vol 42, No 1, pp4250 (1995)

[3] M.I. Haller, \& B.T. Khuri-Yakub, "A Surface Micromachined Electrostatic Ultrasonic Air Transducer" IEEE Transaction on UFFC, 1996. 43(1): p. 1-6

[4] K.S. Ho, R.J. Bradley, D.R. Billson and D.A. Hutchins "Microstereolithography as a transducer design method”, Ultrasonics. 48, 1-5 (2008).

[5] A. Octavio et al "Air-coupled linear and sparse cMUT array manufactured using MUMPs process" Microsyst Technol Vol 17, pp1635-1644 (2011)

[6] R Pericet-Camara, "Microstructuring of Polystyrene Surfaces with Nonsolvent Sessile Droplets” ChemPhysChem Vol 9, pp 1738 - 1746 (2008)

[7] H. Ulmke et al, "Piezoelectric Droplet Generator for the Calibration

[8] of Particle-Sizing Instruments” Chem. Eng. Technol. Vol 24, No 3, pp 265-268, (2001)

[9] G.L. Switzer “A versatile system for stable generation of uniform droplets” Rev. Sci. Instrum. Vol. 62,pp 2765-2771 (1991);

[10] G. Müller \& D.W. Baudrand "Plating on plastics" ISBN 9780852180389 